\title{
CUERPO, SALUD Y AUTOLEGITIMACIÓN EN LA CONTEMPORANEIDAD DE LOS JUEGOS Y LOS BAILES TRADICIONALES (ACERCA DE UNA INVESTIGACIÓN ETNOGRÁFICA EN ASTURIAS)
}

\author{
CARLOS SUARI \\ Doctorando en Antropología \\ Universitat Rovira i Virgili \\ carlos.suari@estudiants.urv.cat \\ https://orcid.org/0000-0002-0575-988X
}

RESUMEN. En este texto se parte del carácter paradójico del enfrentamiento entre la tradición y la contemporaneidad, considerando que el cambio, en sus innumerables manifestaciones, es la única forma de continuidad posible para cualquier práctica cultural. El análisis se detiene aquí en unas transformaciones en concreto. Se trata de las que tienen lugar en la fricción que se registra durante la perpetuación de algunos juegos y bailes tradicionales dentro del contexto fitness global. Sus practicantes, que se han adherido a ellos mediante la indiscutida costumbre familiar o a través de un nuevo compromiso de identificación étnica adquirido individualmente, son emisores y receptores potenciales de inéditos discursos, algunos de los cuales son el centro de esta etnografía. Concretamente, aquellos que como encarnación o como predicación se sitúan en el ámbito del cuerpo y de la salud pero que, no obstante, son asumidos heterogéneamente, pues también traen consigo ostensibles disidencias. Finalmente, se discute el régimen de influencias de lo global sobre lo tradicional en lo que a este trabajo respecta y se extrae en conclusión el papel 
autolegitimador de algunas de estas narrativas habida cuenta de la minorización social de estas prácticas etnomotrices en la actualidad.

PALABRAS CLAVE: globalización; tradición; deporte; cuerpo; salud.

BODY, HEALTH AND SELF-LEGITIMISATION IN MODERN MANIFESTATIONS OF TRADITIONAL GAMES AND DANCES

AвSTRACT. This text begins with the paradoxical nature of the confrontation between tradition and contemporaneity, considering change, in its innumerable manifestations, as the only possible form of continuity for any cultural practice. The analysis then looks at specific transformations relating to the friction that occurs regarding the perpetuation of certain traditional games and dances within the overall context of fitness culture. Their practitioners, who have maintained these practices through unquestioning commitment to family custom or through a new commitment to individually acquired ethnic identity, are potential senders and receivers of new discourses, some of which are at the center of this ethnography. Specifically, the text examines those discourses that, as incarnation or as predication, are situated in the sphere of the body and health, but which, nevertheless, are adopted heterogeneously, since they also bring with them ostensible disagreements. Finally, the article discusses the regime of global influences on the traditional and the selflegitimizing role of some of these narratives, taking into account the social minority of these ethnomotor practices at present.

KEYWORDS: globalization; tradition; sport; body; health. 


\section{Tres posibles paradojas y otros aspectos introductorios}

Antes de desgranar otras cuestiones preliminares, quisiera situar temáticamente este artículo. Las prácticas culturales consideradas tradicionales no son ajenas al cambio, sino que resultan especialmente afectadas por la contemporánea exigencia globalizadora, cuestionadas en su concepción como castizas. Esta expectativa de transformación y resistencia es analizada aquí sobre unas actividades tradicionales concretas: los juegos y los bailes. Ellos estaban (de algún modo) desde mucho antes, pero ahora las técnicas del cuerpo ocioso, ritualizado, lúdico, expresivo, domesticado..., son también (y sobre todo) otras. Sus regímenes hace décadas que contactan, chocan y se influyen desigualmente. Hay quien incluso las emparenta. A un lado, la rayuela y la jota, al otro, el fútbol y la última forma de hacer aeróbic. ¿Quién lo iba a decir? En todo, ahí están, tan lejos y tan cerca.

Lo que me dispongo a plantear es una inmersión en dos aspectos de lo que podría considerarse un interior de los agentes de práctica (y, en consecuencia, también agentes de esa mutación adaptativa de la propia práctica). Me centraré, pues, en las transformaciones corporales y en las programaciones narrativas que, sobre lo higiénico o en apoyo de lo higiénico, se desarrollan ante y durante estas prácticas tradicionales en su problemático acoplamiento en lo global. Pero, antes de nada, quisiera justificar la importancia de haber vislumbrado tres posibles paradojas que contribuyen a jalonar este espacio de investigación.

Las prácticas que comúnmente consideramos, distinguimos o especializamos como tradicionales también forman parte del escenario cultural variablemente globalizado en el que vivimos una gran parte de la población del mundo (Appadurai, 1996). Tanto, que cabría pensar que las «tradicionalizamos», es decir, que las pensamos como tradición en arreglo o reacción a esa vivencia global. En algunas ocasiones estas prácticas resultan desplazadas más o menos abruptamente hacia el olvido definitivo y sin rastro. Otras veces, ciertas muestras materiales o inmateriales de ellas pasan a las vitrinas o a los archivos audiovisuales a modo de testimonio vestigial. Pero quizá donde se hace más patente esa combinación paradójica de las múltiples nociones de tradición y modernidad es en 
aquellas prácticas que de algún modo continúan vivas, mutando con más disimulo o estridencia, en permanente reajuste al escurridizo presente (García Canclini, 1990). Lo tradicional nos remite a lo local, al pueblo (y, por lo tanto, a lo colectivo y a lo étnico), a las ideas de heredar, mantener y transmitir. Ideas que, por cierto, suelen estar vinculadas al peso grave de la memoria legada y a un escrupuloso compromiso de minimización de los cambios. Parece, al contrario, que lo contemporáneo implica la novedad y hace una invocación al surgimiento de lo inédito, en un deseo de atraer y gustar masivamente, aun desde la vivencia más individualista e ilusoriamente original.

En ese indefectible y necesario mudar de las prácticas culturales tradicionales a través del tiempo, aprecio una nueva paradoja discursiva que viene a señalar que este proceso transformativo no adquiere, en absoluto, un único vehículo expresivo. Contrariamente, podría decirse que posee un carácter divergente cuyas formas clasificaré en dos tipos: las encarnaciones y las predicaciones. Unas, las primeras, se refieren a las vivencias incorporadas en y mediante el cuerpo en (o ante) la práctica (Csordas, 1994). Las segundas, en cambio, se manifiestan habitualmente de forma verbal, como norte teleológico y como dispositivo argumental (Fernández McClintock, 1996), tal vez preparadas para la posible discusión derivada del rozamiento tradición-modernidad (Appadurai, 1996; García Canclini, 1990). A pesar de su elocuente diferencia expresiva, en este artículo, unas y otras se agolparán, no obstante, en el mismo dominio, dentro de las lindes del cuerpo.

Quizá esto último, en relación con las impresiones que he venido recogiendo en el campo, abra las puertas a una tercera posible paradoja. Las argumentaciones populares sobre este tipo de fricciones entre prácticas consideradas tradicionales y globalización pareciera que se nutren más de lo material que de lo inmaterial, más de lo envolvente que de lo interior, más de la generalización que de la concreción. Así, por ejemplo, abundan los relatos sobre la variación en los objetos que se manejan en la práctica o sobre la vestimenta (de la que también hablaré aquí), pero es raro que estas narraciones acorten su radio y entren en lo íntimo de las personas practicantes, las personas que, por lo tanto, son las prota- 
gonistas de estas transformaciones culturales que me ocupan. Por otro lado, parece que se han hecho urgentes y hasta efectivas las visiones de conjunto sobre esta transformación, como si el propio humor globalizado exigiera siempre respuestas globales e interpretaciones a gran escala en las que observo una omisión, una desmemoria de lo íntimo.

\section{Etnomotricidad y contexto global}

Como ya he anticipado, son algunas prácticas en concreto en las que me he detenido a observar estas paradojas entre la tradición y la contemporaneidad. Aun confiando en la suficiencia del conocimiento común que permite reconocer y distinguir un baile o un juego tradicional, señalaré que sobre ellos convergen dos cargas semánticas mínimas que posibilitan verlos a ambos en un dominio común que podríamos llamar y se ha llamado cultura física, corporal o motriz o, quizá más etimológica y propiamente, etnomotricidad, lo que para Parlebas (1999: letra e, párrafo 151) es el «champ et nature des pratiques motrices envisagées sous l'angle de leur rapport à la culture et au milieu social au sein desquels elles se sont développées». Sus prácticas constitutivas (Parlebas, 2016b:302) «ont toutes en commun une mise en jeu corporelle qui vaut pour elle-même et non pour un résultat extérieur, comme c'est le cas dans les situations de travail», y vienen a diferenciarse de otras también de tipo motor o deportivo por su enraizamiento «dans une longue tradition culturelle, mais qui n'a pas été consacré par les instances institutionnelles» (Parlebas 1999: letra j, párrafo 72).

Los juegos y los bailes tradicionales son, con toda seguridad, las dos formas de etnomotricidad más relevantes, extendidas o reconocibles dentro de los repertorios de prácticas de cada pueblo. No obstante, en ese mismo recorte etnomotor, estas dos conviven con las danzas, las luchas y cualquier otra manifestación motriz similar en cuanto a haber sido transmitida-aprendida por tradición cultural. En este sentido, la noción de técnica corporal de Mauss (1935: 278), a la que Parlebas ha hecho referencia como una de las pioneras en el estudio sociocultural de la motricidad, resulta absolutamente clarificadora en lo que respecta a la elaboración cultural de la acción motriz: «No hay técnica ni transmisión si no hay tradición». 
Actualmente, en el ámbito académico que se ocupa de la actividad físico-deportiva, la etnomotricidad es una presencia a menudo evitada, semitransparente, incluso a veces molesta, prácticamente liquidada de unos planes de estudio y unos equipos de investigación que presentan, a grandes rasgos, una preferencia creciente por la pedagogía del récord y la tecnificación deportiva, la construcción anatómica, la programación metabólica y otras derivadas biomédicas. A pesar de este lugar residual dentro de la academia, algunas prácticas etnomotrices viven hoy un intenso proceso de contemporaneización, resignificación y reapropiación. En Asturias, como en otros lugares, los juegos y los bailes tradicionales incorporan practicantes incluso en contextos novedosos, fuera de la transmisión familiar o de los ámbitos rurales, que es donde mejor se han conservado dichas manifestaciones.

En la constatación de las variaciones en los detalles más pequeños y anecdóticos, o en la discusión de los grandes fenómenos de carácter cultural y político, la fricción entre motricidades tradicionales y motricidades hegemónicas comenzó a hacerse evidente en las últimas décadas. Así, Jaouen (2016) ha visto en las acciones de salvaguarda y contemporaneización de la motricidad tradicional una reacción al exceso de protagonismo y a la descomunal capacidad de condicionar la vida institucional que trae consigo la consagración del deporte moderno como uniformidad corporal universal. O, un poco más allá, Eichberg (2001) ha asegurado que la recuperación y la proyección a futuro de las prácticas tradicionales contribuye a discutir la noción de pueblo, facilitando la igualación del demos con el ethnos y superando con ello la concepción de la democracia como simple ejercicio representativo con una más compleja de democracia viva, ejercida, participada. Por su lado, During (2016: 74) ha establecido una comparación de ambas motricidades, convencido de que las tradicionales, «plus complexes dans leur déroulement, moins centrés sur le score que sur l'intérêt des rôles et des séquences, ils font passer le plaisir de la pratique avant l'intérêt du spectacle, la participation de tous avant l'exploit de quelques-uns». No obstante, el propio During (op. cit.) ha considerado que es posible imaginar un espacio de convivencia para la motricidad de carácter universal (las disciplinas deportivas modernas) con una motrici- 
dad patrimonial (la de los bailes y los juegos tradicionales) porque estos, «permettant l'affirmation de la diversité des cultures, ils constituent en même temps que la pluralité de langues, toute la richesse d'un patrimoine, ce que ne diminue en rien l'intérêt de disposer d'un langage universel». En esa misma línea que podríamos etiquetar como patrimonialista, entre otros muchos, Parlebas (2016a: 11) ha urgido a proteger estas manifestaciones tradicionales considerando que «sont la vivante mémoire des gestes du terroir» $y$ «témoins des normes et des valeurs de leur communauté»; pero también se han apuntado diferentes dificultades y problemas en la patrimonialización y la institucionalización, en ese paso del «autorreconocimiento» al «heterorreconocimiento», donde «lo popular» también puede mutar en «lo objetual» bajo la amenaza que implica imitar las formas del deporte global o caer en el legislacionismo, el fetichismo y la descontextualización (Suari, 2017; 2019; 2020).

No en vano, la globalización nos presenta unos patrones generales de práctica motriz reconocibles, dominantes, bien arraigados. El fitness, quizá el que dispone el marco más capaz de hospedar a los demás patrones, ha sido entendido (Landa, 2009: 11) como el «sector productivo y de consumo que ofrece al consumidor masivo un conjunto de servicios para su transformación corporal, para su recreación, para su ejercitación, los cuales se asocian con una forma específica de vida activa y saludable». Del mismo modo, Costa (2017: 113) ha coincidido en entender que el fitness no es solo una especialidad deportivizada, sino un dispositivo que ajusta el cuerpo fit, el cuerpo en forma, a unos mandatos de consumo y vida saludable ineludibles. Una vida saludable que, entre otros parámetros, está caracterizada por «la práctica de alguna actividad física (desde deportes tradicionales hasta ejercicios construidos, rutinas aeróbicas y disciplinas como el yoga, el taichí o pilates)». En otra de sus aportaciones, Landa (2012: 229) ha establecido que «[o] sujeito fitness é aquele modelo não só da perfeição estética, mas também, e fundamentalmente, um parâmetro sanitário que prescreve a sua contraparte como patológica». Ortega (2003), situando las prácticas del fitness en la esfera de lo que considera ascético, también ha señalado que el énfasis puesto contemporáneamente en los cuidados corporales, médicos, higiénicos y estéticos 
promueve la formación de bioidentidades, identidades somáticas. Complementariamente, el bodybuilding, devenido ya en un campo especializado del fitness, puede seguir presentándose como una finalidad competitiva (culturismo), tal era en su origen, o como un medio intermedio para la consecución de diversas metas. Así, Kogan (2005) ha planteado que, si antaño eran las personas culturistas quienes buscaban estas metamorfosis anatómicas y funcionales, hoy cualquier practicante de fitness halla en las diferentes maneras de acercarse al bodybuilding una performatividad, una particularización corporal.

Aunque al auspicio del fitness, el sport for all marca un camino de práctica característicamente transversal, multinivel, para toda condición, que lleva las actividades físicas a un lugar de cotidianidad y normalización nunca antes visto, en un régimen de prescripción de las instituciones sanitarias y escolares que cuenta con la publicidad constante de los medios de comunicación. Medina Cano (1998: 118) explica que el deporte es ya un elemento «esencial e inseparable de la vida moderna y del progreso material» que se relaciona con la industria y la sociedad urbana mediante ciertas características: «[...] aplicación racional del tiempo, disciplina y esfuerzo, autoridad, competitividad, resultados cuantificables, objetivos racionales y organización». Al hilo de este carácter deportivizante, destaco otros dos patrones muy usuales en nuestros días. Por un lado, la proliferación de estrategias para la creación y la detección de talentos motores precoces, $y$, por otro, el aumento de las competiciones amateurs en eventos dirigidos a no profesionales de diferentes modalidades de juego, pero también de baile, a personas que acaban compatibilizando sus vidas laborales y familiares con unas formas de práctica enormemente exigentes.

El ejercicio físico y el deporte han sido vistos como una de las cuatro dimensiones de control y consumo del cuerpo (Esteban, 2004: 68), como lugares donde el cuerpo se supedita a la noción de máquina, a la producción, al rendimiento, a la competitividad y a la mercantilización (Brohm, 1992 [1976]), o como ámbitos para el desarrollo de los extremos de domesticación y control anatomopolítico (sobre el cuerpo individual) y biopolítico (sobre el cuerpo especie), tal y como ya expusiera Foucault (1976). Por su lado, en obras como la de Callinicos (1993 [1989]) se ha ma- 
nifestado que las prácticas motrices contemporáneas implican una forma de disciplinar el cuerpo en la dirección de convertirlo en un símbolo de estatus de acuerdo con ciertas ideas de juventud, energía, movilidad o salud. De hecho, Rangel Lara (2020: 310-311) también ha constatado el establecimiento de «ideales que promueven los cuerpos esculpidos y disciplinados», ya no por su posible valor estético, sino porque «el tiempo y los recursos económicos invertidos en esta tarea» pueden interpretarse como un excedente de medios, como una señal de riqueza. Décadas antes, Bourdieu (1979; 1980; 1986) ya había señalado los vínculos entre el cuerpo como marcador de posición social y las nociones de distinción, gusto y habitus, y Douglas (1971: 387) había manifestado que el cuerpo, «as a vehicle of communication, is misunderstood if it is treated as a signal box, a static framework emitting and receiving strictly coded messages. The body communicates information for and from the social system in which it is a part». En diálogo con Douglas y ante los escenarios actuales de transformación corporal, Le Breton (1994: 209) ha señalado que, «si el cuerpo es un símbolo de la sociedad, toda amenaza de su forma afecta simbólicamente al lazo social. Pensar el cuerpo es otra forma de pensar el mundo y el vínculo social; un trastorno introducido en la configuración del cuerpo es un desorden introducido en la coherencia del mundo».

\section{Claves metodológicas}

Tras contextualizarla en algunas miradas y reflexiones sobre la etnomotricidad y el cuerpo en el mundo deportivizado actual, llega el momento de concretar cómo se ha concebido y desarrollado esta contribución. Su finalidad principal es estudiar y describir las variaciones y las permanencias en los discursos sobre el cuerpo y la salud que se están dando en la práctica contemporánea de los juegos y los bailes tradicionales. Relacionar estas nuevas experiencias con las formas de permeación y asunción global de las narrativas científico-técnicas de tipo biomédico ha sido el objetivo direccional en la discusión del material. Hay que señalar que este recorte no es más que uno de los posibles dentro del trabajo marco del que surge el presente artículo y del que, por lo tanto, es deudor metodológico. 
Se trata de un proyecto de tesis doctoral que pretende estudiar antropológicamente la expresión contemporánea de las prácticas etnomotrices asturianas, analizándola desde tres perspectivas complementarias. La primera, estructural y funcionalmente interna, repara en las transformaciones de los rasgos constitutivos de estas prácticas. La segunda se ocupa de sus nuevos significados y contextos. Finalmente, la tercera relaciona el proceso de patrimonialización de estas prácticas (y, por lo tanto, su positivización, estandarización e institucionalización) con los procesos de identificación étnica (lo asturiano) y las (contra)hegemonías culturales.

Aunque el trabajo de campo se ha desplegado sobre todo en Asturias, ciertas experiencias de observación que han tenido lugar en otros escenarios de práctica etnomotriz del suroeste europeo habilitan puntuales reflexiones de conjunto, comparativas concretas. Las personas informantes presentan diferentes formas de vinculación a estas actividades etnomotrices. Algunas han recibido los juegos o los bailes tradicionales por la vía familiar, practicándolos únicamente en estos contextos. Otras, en cambio, se han movido al encuentro de comunidades de práctica, como clubes o asociaciones.

La investigación se ha desarrollado a partir de una concepción longitudinal, dinámica y diacrónica que recoge la elaboración empírica de casi veinte años (2002-2021) de inmersiones en el campo. La observación participante, además de la técnica primordial, ha sido la piedra de toque sobre la que han descansado otras técnicas como la entrevista o los grupos focales que, en cualquier caso, se han pensado como instrumentos de indagación complementaria dentro de una vivencia que ha sido posible desempeñando roles de campo muy diversos y siempre prolongados en el tiempo. En lo que respecta específicamente a este trabajo, algunos de los roles han sido el de jugador, monitor y árbitro en distintas modalidades de juegos tradicionales, el de aprendiz de bailador en diferentes talleres y grupos y el de asistente y participante en diferentes eventos, tanto espontáneos como organizados, sobre la práctica del juego o el baile tradicional. Concretamente, el material aquí manejado proviene de algunas recogidas realizadas en torno a las modalidades de bolos federadas (Mieres 2018 y Cangas del Narcea 2012), un club dedicado a un variado repertorio de jue- 
gos tradicionales mediante acciones de divulgación y competición realizadas por todo el territorio asturiano (Cangues d'Onís 2009); el baile tradicional en contextos espontáneos (Cangas del Narcea 2016) y en contextos formalizados y didactizados (Avilés 2018 y Uviéu 2020), y la convocatoria de talleres y muestras de juego tradicional (Corvera 2010 y Xixón 2017).

\section{Resultados}

La categorización del material es una de las principales decisiones a la hora de presentar los resultados. No solo por la importancia narrativa que implica, sino porque dirige de algún modo la discusión y las conclusiones. En este caso, y en este sentido, las decisiones han sido dos. Por un lado, no dividir necesariamente el material empírico en discursos sobre la salud y discursos sobre el cuerpo. Frecuentemente me he encontrado que la ligazón entre ambos aspectos era muy fuerte, de modo que su separación sería un arreglo metodológico injustificado e imprudente. Al contrario, he ido convenciéndome de la utilidad de clasificar los datos dependiendo de si revelan asunciones o disidencias en relación con los nuevos discursos sobre el cuerpo y la salud. De hecho, esa diferenciación (entre incorporar y rechazar estas formas inéditas de realizar y narrar la práctica) se presenta frecuente, espontánea y expresamente entre las personas del campo, emergiendo así entre ellas una forma de alteridad que considero especialmente descriptiva. Por lo tanto, si bien el escenario de transformación ya podría considerarse explícito al constatar el surgimiento de nuevas corporalidades y narrativas sobre la salud, en su negación, rechazo o discutida aceptación aflora otra forma de confirmar la propia existencia de lo que he convertido en el objeto de estudio principal de esta contribución.

\subsection{Lo asumido}

Una pequeña muestra del verbatim puede dar una idea de la profundidad y la diversidad con las que aparecen hoy en el entorno de los juegos y los bailes algunas ideas sobre la salud y el cuerpo. «Venir por la bolera es muy sano», expresa generalizando una jugadora de cuatriada, la modalidad 
de bolos más practicada en el centro de Asturias. «Endureces y te pone fuerte», comenta un practicante y monitor de ese mismo juego, también en Mieres. «Respiro, me desestreso», señala una compañera con la que coincidí aprendiendo a bailar el repertorio tradicional en los talleres de un grupo cuya actividad se centra en Avilés. «Coges postura», sentencia un monitor de baile tradicional que imparte un taller monográfico sobre la jota al que asistimos más de 30 aprendices. «Estoy adelgazando con esto», explica otra compañera durante una de las clases a las que estuve asistiendo más de un año, semanalmente, en Fitoria (Uviéu). «Aprenden a manejarse en grupo» $\mathrm{y}$ «se adaptan» son dos comentarios que lanza el responsable de una ludoteca tradicional para escolares en Xixón, mientras que una de las alumnas receptoras de la propuesta me explica: «Nos respetamos y somos felices». Las tres dimensiones ya clásicas de la salud (física, psíquica y social) pueden vislumbrarse desde estos y otros recortes similares de mi cuaderno de campo. Esto evidencia que los discursos sobre el potencial salutífero y transformador (mejorante) del cuerpo pueden estar en boca de cualquier persona que, hoy en día, juegue o baile «tradicionalmente» en Asturias.

Sin haber buscado específicamente este tipo de reflexiones (más bien, al contrario, explorando todo tipo de motivaciones, estimaciones y apreciaciones en torno a la práctica), las experiencias y las apreciaciones higiénico-corporales están servidas. El hecho de bailar y jugar (a la manera que se concibe como tradicional) se está explicando desde ciertas argumentaciones autolegitimadoras, tal como si a quienes observo y me informan sintieran la necesidad de subrayar unas propiedades y conveniencias para la salud a través de sus cuerpos. Constato también la progresiva didactización en algunas pautas de práctica y, más escasamente, una incipiente monitorización. Por didactización entiendo la implementación de nuevos sistemas expertos de (auto)instrucción que conllevan la entrada en funcionamiento de ciertos principios metodológicos: calentamiento, entrenamiento, temporalización, calibración de la intensidad, programación de objetivos y medios, etc. Al hablar de monitorización me refiero a la incorporación de ciertos «monitores», es decir, medios tecnológicos que sirven para la medición y el control de la práctica motriz o de 
la fisiología durante esta. Es el caso de los pulsómetros, los dispositivos GPS, los acelerómetros o las aplicaciones informáticas, también las de bolsillo, para la gestión de la didactización de la práctica.

Otro elemento que se asume progresivamente es la gerontización de algunas prácticas, sobre todo de juego, como si se trataran de un «último recurso» de salud o, más bien, de una posibilidad de «mínima salud», fundamentalmente de tipo relacional, a través de algunas formas de motricidad que apenas contrarrestan la herrumbe articular de los años pero que son suficiente pretexto para fomentar la socialización. Así es como ciertos juegos tradicionales, los cuales nunca habían sido percibidos como exclusivos de las edades más avanzadas, van quedando socialmente arrinconados en estos sectores de la población, habida cuenta de una previsión de empobrecimiento de la condición física de las personas mayores. Esto afecta especialmente a algunos juegos de lanzamiento ligero (generalmente de precisión) que han mudado su presentación social hasta ser vistos como «propios» de mayores, dejando de aparecer en las calles, las fiestas populares o las tabernas y pasando a habitar las zonas verdes de los centros gerontológicos y otros lugares especialmente frecuentados por personas ancianas. En el pequeño parque de Cancienes (Corvera), pude detenerme a hablar con un grupo de hombres de más de 70 años que "pasaban el rato» lanzando unas fichas a la llave (modalidad esta, por cierto, que llegó a tener su propia federación en los años noventa y ahora se encuentra absolutamente denostada). Uno de ellos resumía muy bien su experiencia: «Vengo aquí porque te despeja la mente, hablas un poco con la gente y te relajas. A mí el juego me da bastante igual».

El cuerpo que se reforma a través de las maneras contemporáneas de práctica de estos juegos y bailes constituye un ámbito de condensación de experiencias pero también, a su vez, una experiencia independiente. Dicho de otro modo, si bien una nueva forma de corporalidad debe ser entendida como el resultado del entrelazamiento de diversos discursos, también ha de verse como un nuevo discurso en articulación con los ya presentes en la arena de juego o baile correspondiente. La aparición de entrenamientos o ensayos y el aumento de citas de competición o exhibición han elevado la repetitividad con la que hoy aparecen algunas de estas 
actividades tradicionales. Así, el proceso de transformación corporal, en forma de tallado anatómico y adaptación conductual (coordinativa, gestual), se adapta a esta insólita intensificación que se acompaña de una exótica tecnificación del disciplinamiento. Por lo tanto, del mismo modo que puede distinguirse un cuerpo futbolista o ciclista, comienza a ser posible reconocer los cuerpos que bailan o juegan tradicionalmente. Esta nueva regulación corporal, distinta a la que se podía conocer hasta ahora, no ha de entenderse como un paso de la naturalidad a la artificialidad o del salvajismo a la disciplina. Se trata, en todo caso, de una nueva forma de artificialidad disciplinada a base de más horas que nunca y a través de maneras de aproximación a la práctica que han aparecido recientemente en estas comunidades lúdicas. De ello deviene un discurso motor y corporal nuevo, nuevos rasgos posturales y nuevas conductas motrices: «Con los bolos también haces cuerpo, sobre todo si entrenas para competir», recojo a un destacado jugador en una bolera de Cangas del Narcea.

En la vestimenta, entendida como cercanía y primera extensión del cuerpo, también he reconocido un escenario de transformación en relación con las expresiones actuales de los juegos y los bailes tradicionales. Por un lado, he apreciado un debate en cuanto al uso del uniforme en el desarrollo de estas actividades. Así, en algunas modalidades de juego ya claramente deportivizado la entrada del uniforme ha sido dispar. Por un lado, desde un cierto romanticismo y una notable creatividad, se han planteado uniformes deportivos con referencias a la indumentaria reconocida popularmente como tradicional (fundamentalmente, esquemas de vestimenta del siglo xix). Por otro, también se ha defendido una uniformidad neopaisana (es una licencia calificativa propia) a base de camisas y pantalones o faldas de acuerdo con lo que pudo ser el vestir corriente hasta la llegada masiva del chándal y de otras prendas deportivas. Pero, a fin de cuentas, parece que dentro de las prácticas deportivas tradicionales el atuendo que gana terreno es este último, el de las llamadas prendas técnicas, sintéticas, transpirables: camisetas, polos, pantalones cortos y largos, zapatillas que se venden como de atletismo, tenis o balonmano. En el ámbito del baile tradicional, el uso de la indumentaria tradicional tiene sin duda una mayor adherencia. Pese a ello, no han remitido los 
debates sobre hasta qué punto es necesaria esta asociación o cuáles deberían ser sus limitaciones o principios. Consecuentemente, la discusión sobre la autenticidad de la recreación de esas vestimentas tradicionales sigue también abierta y es motivo de no pocas preocupaciones y esmeros de quienes bailan el repertorio asturiano, especialmente de cara a exhibiciones y competiciones. Aparecen, no obstante, cada vez más colectivos y personas que sienten la necesidad de independizar la práctica coreográfica del uso del traje tradicional e incluso parece que han aumentado las oportunidades de ver bailar con ropa de calle ya no en manifestaciones más o menos espontáneas, sino en actuaciones e incluso en alguna representación formalizada de tipo didáctico.

Cabe destacar que ciertas concepciones biomecanicistas y ergonomistas también han aparecido en el contexto del cuerpo vestido para jugar y bailar tradicional. Así, por ejemplo, he podido recoger diferentes justificaciones sobre la conveniencia del vestir tradicional para el baile basándose en que, con él, la expresividad es mayor y se alcanza un total desarrollo de los movimientos. También aparecen en el campo ciertas reflexiones sobre la modulación y el ajuste en el vestir buscando un difícil convenio entre la dimensión estrictamente estética y la prevención de daños o la optimización del rendimiento físico. Una de estas reflexiones fue clave para que en algunas modalidades de juego deportificadas a principios de los años dos mil dejaran de utilizarse los uniformes a los que antes me refería como neopaisanos, aquellos que se habían inspirado en el vestir inmediatamente anterior a la llegada de la moda deportiva. La elasticidad, frescura, ligereza y libertad de movimientos de la camiseta acabaron por desplazar a los botones y los cuellos de las camisas, mientras que los cordones del chándal aportaban un ajuste más cómodo que las hebillas de los cinturones.

\subsection{Lo disidente}

El rechazo, la puesta en duda o la asunción parcial o irregular de los nuevos discursos sobre el cuerpo y la salud en el ámbito de los bailes y los juegos tradicionales suponen, como ya he señalado anteriormente, un tipo de alteridad interna (dentro de las comunidades de práctica de estas 
actividades) que me parece especialmente útil en la constatación de este fenómeno activo de transformación en las prácticas etnomotrices. Así, un número importante de practicantes se mantiene fiel a las formas de domesticación y optimización previas a los procesos de institucionalización, patrimonialización y deportificación. Ante algunas aseveraciones recogidas en el campo del tipo «ahora es cuando estos juegos se están culturizando», es necesario insistir en la idea de que la construcción cultural de la motricidad (Mauss, 1935; Parlebas, 1999) es inherente a la propia idea de motricidad y, por lo tanto, no debe atribuirse a estos últimos procesos de positivización. Lo que aquí debemos constatar es una coyuntura de cambio en los discursos higiénico-corporales que no implica una ruptura definitiva con los discursos anteriores. Del mismo modo que unas personas se abren a las nuevas formas de abordar el actuar motor en el juego y el baile, otras conservan los patrones aprendidos en su entorno más próximo y otras más hibridan ambas formas de jugar o bailar.

Dentro de las disidencias, hay que destacar una recurrente apelación al carácter diferenciado de estas prácticas tradicionales, las cuales, entienden algunas personas, habrían de permanecer al margen de las influencias de otras formas motrices prototípicamente globalizadas y de moda. Entre estas argumentaciones que podría tildar de diferencialistas, son frecuentes las referencias a la libertad y el desembarazo como sentimientos propios del bailar y el jugar tradicional y no así, a su modo de ver, de otras modalidades. En esta línea, algunas compañeras de clase de baile tradicional en Avilés y Fitoria (Uviéu) me han trasladado sus mayores sentimientos de tranquilidad, desinhibición y seguridad en este contexto que en otros que podrían considerarse similares (también de aprendizaje grupal coreográfico, pero en gimnasios y polideportivos, donde los bailes eran aeróbicos y de salón). Opinaban también que la diversidad corporal era mayor en el ámbito del baile tradicional y que la aceptación y el disfrute de dicha diversidad también eran claramente superiores en contraste con los otros tipos de clases de baile. Parece evidente que esta percepción positiva, por un lado, fomenta la adhesión a esta manera de realizar y sentir la práctica motriz, vinculada a lo tradicional y opuesta de algún modo a lo hegemónico. En buena lógica, las personas que así lo 
sienten rechazan cualquier permeación que pudiera alterar este régimen de pluralidad, libertad y disfrute.

No obstante, la convivencia y la hibridación de las diferentes normatividades corporales se manifiesta de forma exuberante en el campo. Durante una competición en Cangues d'Onís, en un mismo equipo de tiro de soga, un juego colectivo de enfrentamiento, es fácil apreciar diferentes formas de presentarse y de disponerse corporalmente. Así lo confirma la conversación con los protagonistas. Conviven quienes se entrenan con arreglo a las técnicas hegemónicas derivadas del bodybuilding y las investigaciones fisiológicas con quienes mantienen su fortaleza gracias a perseverar en las formas de trabajo y cuidado corporal de toda la vida; quienes llevan la uniformidad del atuendo a un extremo escrupuloso con quienes apenas cumplen con el mandato reglamentario al respetar solamente el hecho mínimo de llevar el mismo modelo de camiseta que sus compañeros. Desde una observación multisituada, también es perfectamente posible contemplar, a día de hoy, modos de práctica diferentes a pesar de estar protagonizados por el mismo juego o baile. Así, en un lugar y momento podría verse bailar a lo llixero (una forma concreta de baile tradicional) de una manera espontánea, vistiendo el atuendo corriente y sin una preocupación especial por cumplir un patrón técnico determinado, mientras que, simultáneamente, la misma modalidad podría estar apareciendo de un modo absolutamente coreografiado, con una técnica depurada y fiel a algún patrón tradicional estudiado junto con una preciosa y milimétrica reproducción del traje del lugar, también de acuerdo con algún modelo establecido. Entre ambos extremos, toda manifestación híbrida o intermedia es posible y compatible.

Otra disidencia sobre lo higiénico que se presenta en el campo constantemente tiene que ver con la continuidad de los consumos tóxicos durante la práctica, a pesar de las recomendaciones o, incluso, las reglamentaciones que tratan de limitarlo. Beber alcohol o fumar son hábitos que tradicionalmente no han resultado incompatibles con el baile o el juego pero que, actualmente, a veces se encuentran perseguidos por las normativas que afectan a determinadas instalaciones, por los estatutos internos de ciertas prácticas o, como resultante multidiscursiva, por una nueva 
conciencia que también ha ido extendiéndose aun en ausencia de sanción. En ese sentido, la fricción es evidente y mientras ciertas personas manifiestan abiertamente su voluntad de no cumplir estos nuevos preceptos justificando que van contra el sentido tradicional de la práctica, otras buscan espacios y momentos liminales para no renunciar totalmente a estos hábitos. La sidra, a todo esto, parece gozar de una cierta bula, escurriéndose con mayor facilidad tanto del marco punitivo como de las nuevas programaciones éticas. Las razones, a buen seguro complejas, exceden el cometido de esta contribución.

También hay personas que critican y se niegan a algunas de las nuevas regularizaciones higiénico-deportivas que aparecen en este ámbito muy en relación con la creciente didactización y monitorización de la práctica que ya he comentado. No es extraño encontrar apreciaciones contrarias e incluso irónicas con quienes sí asumen estos nuevos discursos con respecto al calentamiento, el entrenamiento $u$ otras pautas de salud y rendimiento que en principio favorecerían globalmente al desempeño de la actividad. "Ahora ya los hay que andan mirando las pulseras esas que miden los pasos y el corazón», me comentaba un bailador respecto a algunas personas que hacen uso de los llamados smartwatches. No obstante, es sencillo constatar que las formas de práctica predominantes siguen siendo las mismas que pueden verse en las competiciones, actuaciones o momentos de actividad real, puesto que ni en el baile ni en el juego tradicional han proliferado demasiado los ejercicios preparatorios y en la mayor parte de los casos sigue vigente la máxima popular que indica que a bailar se aprende bailando y a jugar se aprende jugando. Solo algunas actividades deportificadas o ciertas exhibiciones se preparan auxiliarmente añadiendo a los formatos reales de práctica algunas propuestas pedagógicas analíticas, es decir, que trabajan separada y profundamente aquellos puntos que mejorar cultivándolos fuera del aspecto global del juego o el baile en cuestión.

En general, y finalmente, ha de reseñarse que a este ámbito de disidencias de los nuevos discursos higiénico-corporales podrían inscribirse otro tipo de contradicciones, ambigüedades o hibridaciones biográficas, que tal vez no afectan a los procesos de ejecución vistos por separado, 
sino a su congruencia. «Por semana hago ayuno intermitente para definir y también como muy bien. Voy al dietista una vez al mes, que también me controla los recovery y la suplementación. Al fisio voy una semana sí y otra no. Los entrenos los voy apuntando en una libretita, las boladas que hago, los kilómetros, los pesos que muevo y las repeticiones». Son las palabras de un jugador en el contexto de una animosa sobremesa, tras la abundante merienda, también en alcohol, después de un partido. En este juego, deportificado hace algunas décadas, sigue manteniéndose la costumbre que obliga al equipo local a invitar al visitante a mesa y mantel tras la competición. Mi interlocutor me explica sus pautas con toda franqueza y un punto de orgullo, pero varios compañeros y adversarios (en este momento realmente son todos compañeros) se mofan, aunque sin acritud, vanagloriándose de mantener otros hábitos totalmente diferentes, «los de toda la vida»: ningún entrenamiento complementario al propio juego, ninguna monitorización, ningún control nutricional. Ambas posiciones se extrañan mutuamente pero también se conocen. Todos saben cómo se come y se vive en sus casas y pueblos sin arreglo a la competición, pero muchos de ellos ambicionan mejorar sus resultados y quizá tener un físico más joven y vigoroso. Me da la sensación de que en este momento, en el que todos consumen grandes cantidades de comida y vino (después llegan los destilados), mi entrevistado está disfrutando de su cheat day: una vez jugado el partido y tras una semana de esfuerzos, la velada poscompetitiva, gastronómica y de camaradería es una posible recompensa. Mientras anochece, encienden sus cigarrillos y se dirigen nuevamente a la bolera. Ahora mano a mano, pues los equipos hace tiempo que se han diluido, el deporte se abate y resurge el juego. Mientras no se haga noche cerrada, la raquítica bombilla será suficiente. En la cantina aneja hay alguna botella de alcohol y latas de refresco y, en un arcón congelador bajo la barra, un paquete de cubitos de hielo.

\section{Conclusiones}

En este artículo he intentado presentar las transformaciones en marcha, en lo que a las experiencias corporales y de salud se refiere, dentro del ámbito del juego y el baile tradicional. No obstante, entre quienes, en un 
extremo, desarrollan una total asunción de estos nuevos discursos y quienes, en el otro, los rechazan de plano, existen un sinfín de posibilidades intermedias que implican todo tipo de formas de hibridación entre los modelos de práctica tradicionales y las nuevas mundologías. Dado que la incorporación de estos discursos aún es discontinua, he hecho hincapié en las alteridades entre quienes asumen y rechazan cada una de estas nuevas formas de corporalidad y salud, alteridades que, lejos de suponer una puesta en duda del proceso de cambio, vienen a confirmar la fuerza con la que se está impactando en estos ámbitos de práctica. Por así decirlo, todas estas constataciones, las cuales voy a presentar a continuación en tres grandes clases que se podrían situar en algún lugar entre la encarnación más estricta (por ejemplo, la variación anatómica) y la predicación puramente moral y verbal (como cuando se asegura que bailar estas especialidades es bueno para la salud), están indisolublemente unidas, anunciando y formando parte de una gran coyuntura de cambio discursivo global.

En primer lugar, he apuntado una progresiva remodelación de los cuerpos hacia una especificidad morfológica y funcional nunca antes vista. Esta nueva regularización corporal obedece a la intensificación de la práctica solo en algunas especialidades, las más expuestas socialmente, bien por el aumento de la frecuencia de exhibición o competición, bien por la aparición de inéditas estrategias de preparación adicionales, o bien por la agregación de ambas. Además, me ha parecido importante considerar estas nuevas presentaciones corporales, esta profunda e inédita encarnación (Csordas, 1994), no solo ya como resultante sino también como un elemento (nuevo) que debe ser analizado en sí mismo y en su articulación con cuantos ya estaban situados dentro y alrededor de cualquiera de las prácticas lúdicas tradicionales. Esta primera clase de constataciones apuntaría a un encuadre global(izado): el bodybuilding como evidencia utilitaria y simbolizante del fitness.

En segundo lugar, se hace patente una progresiva higienización de la práctica con arreglo a ciertas claves metodológicas y científico-técnicas que han ido permeando hacia estas actividades, habría que señalar que indirectamente, puesto que no se conocen investigaciones o aplicaciones 
desarrolladas propiamente desde estas modalidades. Por lo tanto, esta filtración estaría haciéndose posible en la medida en que los discursos biomédicos se popularizan y se vuelven cotidianos en nuestros días, como probablemente en muchas otras de su entorno, en el seno de la sociedad asturiana. En este sentido, cabe recordar que, además de la llamada cultura fitness, la idea del sport for all nutre de elementos conceptuales y tecnológicos a la población en general, divulgando algunos saberes y consumos que inicialmente eran minoritarios. En gran medida, la didactización (bien en cuanto a pautas generales o bien como métodos más estrictos y expertos) y la monitorización (a través de medios más o menos rabiosamente tecnológicos) son deudoras de la implantación del fitness y el sport for all, que, es necesario recordar, han encontrado diversas formas de fomento en los sistemas educativos oficiales y en los medios de comunicación.

Finalmente, en tercer lugar, puedo distinguir un nuevo género de narrativas que, sobre estas prácticas, elaboran las propias personas implicadas en ellas, modificando ostensiblemente la forma de argumentar los motivos que les empujan a tomar parte. Se habla de cosas que no se hablaban antes. Se buscan propiedades y conveniencias corporales y sanitarias. Bailar y jugar tradicionalmente se presenta ahora, también, como una oportunidad salutífera y como un campo de rendimiento en el que pueden obtenerse mejores resultados si se aplican protocolos de entrenamiento y de control biomédico. Nuevamente el fitness, con su sportfor all como guion, parece una buena base moral en la que apoyar una creciente tendencia a la prescripción indiscriminada y cada vez más genérica del deporte. Este, convertido ya en un archilexema de gimnasia, actividad física, ejercicio, juego, baile, paseo o desahogo, anuncia la llegada de todo tipo de virtudes: desarrollo infantil, rendimiento intelectual, antídoto para el estrés, estimulador de la respuesta inmunitaria, conservador del sistema locomotor, ahuyentador de accidentes vasculares..., o, nada más y nada menos, camino hacia la felicidad.

A la vista de estas predicaciones, recupero la lectura que de ellas desarrolló Fernández McClintock (1996), pues, aunque quizá en este caso lo predicado (una condición higiénico-corporal por alcanzar) es más prosai- 
co y argumentativo que tropológico o poético, considero que no deja de existir una búsqueda de sentido objetivante sobre una realidad del todo subjetiva. Y es que, ante su progresiva minorización y desplazamiento a los márgenes de la disonancia con lo hegemónico, ¿qué mejor manera de reivindicarse practicante de un juego o un baile tradicional asturiano que reconociendo en ellos fuentes de salud y mejora corporal? ¿Qué objetivación más potente que la conquista de una vida duradera y saludable y un cuerpo rabiosamente contemporáneo para unas prácticas a las que se llega mediante identificaciones tan subjetivas como las étnicas? Recuperando la idea sobre cultivar el cuerpo más como símbolo de estatus que como objeto (Callinicos, 1989 [1993]), he de decir que no he apreciado en este campo un hipotético esteticismo generalizado en quienes encarnan estos discursos en su propia corporalidad; más bien considero que se trata de una forma incorporada, inscrita en el cuerpo y en sus hábitos, de expresar, proyectar, performar y, en definitiva, defender en primerísima persona esas nuevas nociones higiénico-corporales como algo autolegitimador, que eleva el estatus de quien decide jugar o bailar tradicionalmente en pleno siglo xxi en un auténtico «sur del norte» como es Asturias.

Como ya he reiterado, en absoluto constato que estos cambios se hayan completado dando lugar a unas nuevas encarnaciones y predicaciones homogéneamente asumidas en ruptura con otras disposiciones corporales e higiénicas. La colisión de las convenciones de la experiencia tradicional (que también es eminentemente rural) con las del modelo global contemporáneo da lugar a alteridades en forma de negación e hibridación que son discontinuas. Con esa misma discontinuidad, las argumentaciones de autolegitimación aparecen como una incipiente necesidad expresiva de algunas personas que juegan y bailan a la manera considerada tradicional. Quizá, una manifestación elocuente de la necesidad de justificar la articulación de lo heterogéneo en lo homogéneo, de lo que se alteriza. En todo caso, estas justificaciones vienen a modificar el papel presente y futuro de estas prácticas, a resignificarlas de hecho. Allí donde no había porqués, sino un encaje espacio-temporal heredado y unas finalidades metaculturales indiscutidas para estos juegos y bailes, penetra ahora un compendio de salutíferas propiedades en sintonía con 
las que se enuncian frecuentemente desde algunos efectores de poder como las marcas deportivas, los colegios médicos o el currículum escolar. Queda en abierta disputa la guisa de reproducción presente y futura de estas prácticas tradicionales, esas que de algún modo sus agentes se han resistido a abandonar. ¿Acabarán haciéndose fuertes en las disposiciones propias o asumirán, al contrario, las apariencias y las argumentaciones de la hegemonía global? La predicción antropológica no es arriesgada ni certera. A pesar de las hipotéticas aspiraciones de quien pudiera operar desde algún tipo de fundamentalismo, la respuesta probablemente estará en algún grado de la fricción hibridante.

\section{Bibliografía}

Appadurai, A. (1996). Modernity at Large: Cultural Dimensions of Globalization. Minneapolis: University of Minnesota.

- (1979). La distinction critique sociale du jugement. Paris: Éditions de Minuit.

- (1980). Le sens pratique. Paris: Éditions de Minuit.

- (1986). «Notas provisionales sobre la percepción social del cuerpo». En VV. AA. Materiales de sociología crítica (pp. 183-194). Madrid: La Piqueta. Brohm, J. M. (1992). Sociologie politique du sport. Nancy: PUN. (Reedición del original de 1976).

Callinicos, A. (1993). Contra el postmodernismo. Una crítica marxista. Bogotá: El Áncora Editores. (Reedición del original de 1989).

Costa, F. (2017). «Vida saludable, fitness y capital humano». En F. Costa, F. y P. Rodríguez (comps.). La salud inalcanzable. Biopolítica molecular y medicalización de la vida cotidiana (pp. 113-139). Buenos Aires: Eudeba.

Csordas, T. J. (ed.) (1994). Embodiment and experience. The existential ground of culture and self. Cambridge: Cambridge University Press.

Douglas, M. (1971). «Do dogs laugh». A cross cultural approach to body symbolism. Joumal of Psychosomatic Research, 15, 387-390.

During, B. (2016). «Jeux, valeurs et cultures en Occident». En P. Parlebas (dir.). Jeux traditionnels, sports et patrimoine culturel. Cultures et éducation (pp. 61-76). Paris: L'Harmattan. 
Eichberg, H. (2001). «L'identité populaire dans le sport et la culture. A propos de la démocratie vivante». En J. J. BARREAu y G. JAOUen (coords.). Les jeux traditionnels en Europe. Education, Culture et Société au xxie siècle (pp. 247-277). Saint Thonan: Éditions Confédération FALSAB.

Esteban, M. (2004). Antropología del cuerpo. Género, itinerarios corporales, identidad y cambio. Barcelona: Edicions Bellaterra.

Fernández McClintock, J. (1996). Campos léxicos y vida cultura n'Asturies. Uviéu: Academia de la Llingua Asturiana.

Foucault, M. (1976). Histoire de la sexualité, 1. La volonté de savoir. Paris: Gallimard.

GarCía CANCLInI, N. (1990). Culturas híbridas. Estrategias para entrar y salir en la modernidad. México DF: Editorial Grijalbo.

JaOUEN, G. (2016).« Jeux traditionnels d'adultes et environnements institutionnels». En P. PARLEBAS (dir.). Jeux traditionnels, sports et patrimoine culturel. Cultures et éducation (pp. 39-69). Paris: L'Harmattan.

KogAN, L. (2005). «Performar para seguir performando: la cultura fitnes». Anthropologica, 23 (23), 153-166.

LANDA, M. I. (2009). «Subjetividades y consumos corporales: Un análisis de las prácticas del fitness en España y Argentina». Razón y palabra, 69. Disponible en: <https://dialnet.unirioja.es/servlet/articulo?codigo=3102757> (consultado: 25 de marzo de 2021).

- (2012). «Os corpos da liderança: as tramas da ficção do dispositivo cultural do fitness». Caderno Cedes, 32 (87), 223-233.

LE BRETON, D. (1994). «Lo imaginario del cuerpo en la tecnociencia». Reis, 68/94, 197-210.

Mauss, M. (1935). «Les techniques du corps». Journal de Psychologie Normale et Pathologique, 271-293.

Medina Cano, F. (1996). «El cuerpo en la posmodernidad: la salud, el ejercicio físico y el cuerpo perfecto». Signo y Pensamiento, 28 (XV), 99-120.

Ortega, F. (2003). «Práticas de ascese corporal e constituição de bioidentidades». Cadernos Saúde Coletiva, 11 (1), 59-77.

Parlebas, P. (1999) Jeux, sports et sociétés: Lexique de praxéologie motrice. Paris: INSEP Éditions. Disponible en: <https://doi.org/10.4000/books. insep.1067> (consultado: 25 de marzo de 2021). 
Parlebas, P. (2016a). «Préface». En P. Parlebas (dir.). Jeux traditionnels, sports et patrimoine culturel. Cultures et éducation (pp. 11-12). Paris: L'Harmattan.

- (2016b). «Réflexions finales: un passé qui ouvre sur l'avenir». En P. PARLEBAS (dir.). Jeux traditionnels, sports et patrimoine culturel. Cultures et éducation (pp. 293-304). Paris: L'Harmattan.

Rangel Lara, T. (2020). El discurso fitness hecho cuerpo. Revista Ciencias y Humanidades, 11 (11). Disponible en: https://revistacienciasyhumanidades.com/index.php/home/article/view/162 (consultado: 25 de marzo de 2021).

SUARI, C. (2017). «Alrededor de la institucionalización de los xuegos tradicionales: de la necesidá de conservalos al peligru de deformalos». Cultures. Revista Asturiana de Cultura, 21, 55-73. Disponible en: <http://academiadelallingua.com/cultures/index.php?px=articulu\&cod=1005> (consultado: 25 de marzo de 2021).

- (2019). «Le jeu traditionnel comme patrimoine à activer. Quelques réflexions à partir du cas asturien». En C. SUARI (dir.). Le jeu traditionnel aujourd'hui. Vigilance, projection et utilité sociale (pp. 11-20). Oviedo/ Uviéu: Asociación del Bolo Vaqueiro \& Sociedá Etnomotora Asturiana.

- (2020). «Anuncies, víes y tresformaciones patrimoniales na contemporaneidá del xuegu tradicional» Cuadiernu. Revista internacional de patrimonio, museología social, memoria y territorio, 8, 89-121. 